\title{
Association between Genetic and Immunological Background of Hepatocellular Carcinoma and Expression of Programmed Cell Death-1
}

\author{
Naoshi Nishida Kazuko Sakai Masahiro Morita Tomoko Aoki \\ Masahiro Takita Satoru Hagiwara Yoriaki Komeda Mamoru Takenaka \\ Yasunori Minami Hiroshi Ida Kazuomi Ueshima Kazuto Nishio \\ Masatoshi Kudo \\ Department of Gastroenterology and Hepatology, Kindai University Faculty of \\ Medicine, Osaka-Sayama, Japan; Department of Genome Biology, Kindai University \\ Faculty of Medicine, Osaka-Sayama, Japan
}

\section{Keywords}

Hepatocellular carcinoma - Immune checkpoint inhibitors · Cancer stem cell · Mutation · Cell signal

\begin{abstract}
Background and Aim: Immune checkpoint inhibitors are promising agents for the treatment of hepatocellular carcinomas (HCC) refractory to conventional therapies. To enhance the efficacy of this treatment, immunological and molecular characteristics of HCC with programmed cell death ligand 1 (PD-L1) should be explored. Methods: Clinical backgrounds, PD-L1 expression, and the amount of CD8+ tumor-infiltrating mononuclear cells (TIMCs) were analyzed in 154 HCCs. The expression of 3 stem cell markers and co-inhibitory receptors on tumor cells and TIMCs, respectively, were examined by immunohistochemical analysis. Somatic mutations in the 409 cancer-associated genes and TERT promoter were determined; HCCs were classified based on the presence of gene alterations affecting the 8 oncogenic pathways. The results were validated using the dataset from the Cancer Genome Atlas. Results: The expression of PD-L1 in the HCCs was positively correlated with progressive tumor features, the presence of cytokeratin 19 (CK19), Sal-like protein 4 (SALL4), and the mutations of genes involving the phosphatidyl inositol 3-kinase (PI3K)-Akt pathway. Although CD8+ cells were densely infiltrated in PD-L1-positive tumors, these TIMCs frequently expressed multiple co-inhibitory receptors. However, a subset of PD-L1-positive tumors characterized by activating mutations of
\end{abstract}


the PI3K-Akt pathway showed a low degree of TIMCs. Conversely, PD-L1-negative HCCs were associated with mutations in the $\beta$-catenin pathway and a small number of TIMCs, although the expression of co-inhibitory receptors was rare. Conclusions: PD-L1-positive HCCs frequently showed an inflamed phenotype with stem cell features; a subset of PD-L1-positive HCCs with mutations in the PI3K-Akt pathway showed a non-inflamed phenotype. In HCCs with dense infiltration of TIMCs, CD8+ cells expressed multiple co-inhibitory receptors, suggesting T cell exhaustion. On the other hand, PD-L1-negative HCCs showed mutations leading to $\beta$-catenin activation and exhibited a non-inflamed background. These characteristics should be taken into consideration for developing novel combination therapies using immune checkpoint inhibitors.

(c) 2020 The Author(s)

Published by S. Karger AG, Basel

\section{Introduction}

Hepatocellular carcinoma (HCC) is the most prevalent primary liver cancer and remains one of the leading causes of cancer deaths worldwide [1]. Recent advancements in the treatment of HCC have improved patient survival; some HCC cases are still treatment-resistant and have an unfavorable prognosis [1,2]. For example, the expression of stem cell markers in HCC is associated with characteristics of advanced tumors such as a high incidence of portal vein invasion, extrahepatic metastasis, and early tumor relapse [3-5]. Although several studies have reported promising molecular targeted therapies for the treatment of advanced HCC, the accumulation of genetic and epigenetic changes in cancer cells may lead to the acquisition of resistance [6-8]. Therefore, it is necessary to develop other treatments to overcome this barrier.

Recently, several studies have demonstrated the efficacy of immune checkpoint inhibitors in HCC, even in tumors that are resistant to tyrosine kinase inhibitors (TKIs). A phase $1 / 2$ clinical trial using the anti-PD-1 (programmed cell death 1 ) antibody nivolumab demonstrated an objective response rate (ORR) of $21-23 \%$ and a disease control rate (DCR) of $58-62 \%$ in patients with advanced HCC [9]. Another phase 2 trial using the anti-PD-1 antibody pembrolizumab showed an ORR of $17 \%$ and a DCR of $62 \%$ in patients who had previously been treated with sorafenib [10]. These data suggest that immune checkpoint inhibitors are a promising option for the management of HCC, especially in patients with progressive disease on TKIs [11]. However, almost $80 \%$ of the patients with HCC do not respond effectively to anti-PD-1 monotherapy $[9,11,10]$. Additionally, although the expression of PD ligand 1 (PD-L1) can be an informative biomarker for effective anti-PD-1 treatment in some tumor types [12], it did not inform on treatment response in patients with HCC [9]. From this point of view, analyzing the immunological microenvironment of HCC is crucial since the response to anti-PD-1 therapy may be determined by the immunological status of the tumor [13]. In addition, as combination therapy using immune checkpoint inhibitors may be required to enhance the antitumor effect, the mutational landscape of PD-L1-positive HCC should be clarified for the development of effective combinations using anti-PD-1 and molecular targeted agents.

In this study, we found a unique immunological and mutational status in the context of the expression of PD-L1 in HCCs. In addition, our data also indicate that refractory HCC expressing stem cell markers may be a good candidate for treatment with immune checkpoint inhibitors.

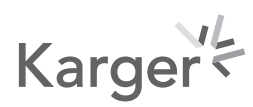




\section{Material and Methods}

Patients

In this study, 154 HCCs and their noncancerous liver tissue counterparts were analyzed. The details of the clinical background of the patients are shown in online supplementary Table S1 (for all online suppl. material, see www.karger.com/doi/10.1159/000506352). The median maximal tumor size in this cohort was $3.85 \mathrm{~cm}$ (distribution: 1.0-15.0). Portal vein invasion was present in 44 HCCs and was not present in 110 HCCs. Thirty-five tumors were well differentiated, 103 were moderately differentiated, and 16 were poorly differentiated.

\section{Immunohistochemistry}

The antibodies and conditions of immunohistochemistry (IHC) used in this study and the cut-off values for estimation of staining in each IHC study are listed in the online supplementary Materials and Methods. Details of the IHC have been previously described [14-18]. For the evaluation of the presence of stem/biliary cell markers in HCC, we examined the expression of epithelial cell adhesion molecule (EpCAM), cytokeratin 19 (CK19), and Sal-like protein 4 (SALL4). For the expression of co-inhibitory receptors on tumor-infiltrating mononuclear cells (TIMCs), we analyzed PD-1, T cell immunoglobulin and mucin domain 3 (TIM-3), and lymphocyte-activation gene 3 (LAG-3) using IHC, and counted the TIMCs that expressed these co-inhibitory receptors. We also analyzed concomitant staining of CD8, PD-1, TIM-3, and LAG-3 to confirm the expression of co-inhibitory receptors on T cells using fluorescence IHC. The details are also shown in online supplementary Materials and Methods.

\section{Mutational Analyses of HCC}

The 154 HCC and liver tissues were stored at $-80^{\circ} \mathrm{C}$ until DNA could be extracted (Kindai cohort). A comprehensive mutational analysis of known cancer driver genes was performed using the Ion AmpliSeq ${ }^{\mathrm{TM}}$ comprehensive cancer panel that covers somatic mutations across 409 cancer-associated genes (Illumina ${ }^{\circledR}$, San Diego, CA, USA). We also analyzed the mutation on the telomerase reverse transcriptase (TERT) promoter [19]. For the association between PD-L1 expression and genetic alterations of each signaling pathway, we also refer to a publicly available dataset composed of 373 HCC cases from the Cancer Genome Atlas (TCGA, provisional). The details are described in the online supplementary Materials and Methods.

\section{Statistics}

We used Pearson's $\chi^{2}$ test or Fisher's exact test for the comparison of categorical variables and the Wilcoxon rank-sum test and Student's $t$ test for continuous variables. To compare the effect of the genetic alterations on PD-L1 expression in the TGCA cohort, we applied logistic regression analysis. All $p$ values were two-sided, and $p<0.05$ was considered statistically significant. All statistical analyses were conducted using the JMP v13 software (SAS Institute Inc., Cary, NC, USA).

\section{Results}

\section{Clinicopathological Features of PD-L1-Positive HCC}

We examined the association between PD-L1 expression in HCCs and the clinicopathological background of the patients. Expression of PD-L1 in HCCs was determined by IHC, which demonstrated that 40/154 tumors (26.0\%) were PD-L1-positive (Fig. 1a). Consistent with a previous study, we found that the expression of PD-L1 was positively correlated with several clinicopathological factors related to the progressive characteristics of HCC, e.g., the presence of vascular invasion, moderate to poor differentiation, and high serum $\alpha$-fetoprotein (AFP) ( $p=0.0021,0.0389$, and 0.0454, respectively; online suppl. Table S2).

\section{Frequent Expression of PD-L1 in Stem/Biliary Cell Marker-Positive HCC}

Expression of PD-L1 in HCCs is associated with high-grade tumors, which are more aggressive. Therefore, we compared the presence of stem/biliary cell markers in PD-L1-positive and PD-L1-negative tumors. Expression of EpCAM, CK19, and SALL4 was found in 60, 30, 


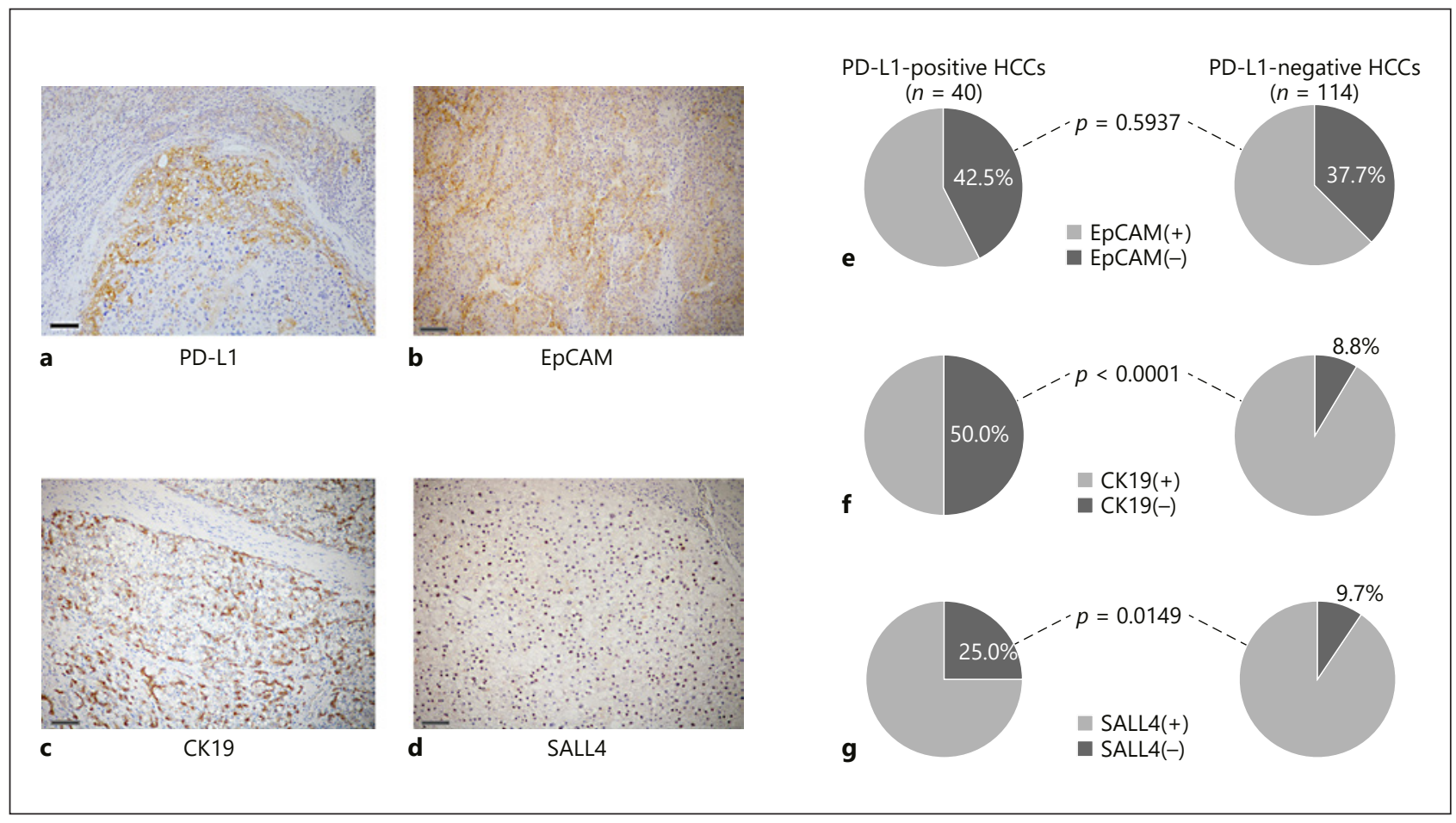

Fig. 1. Association between expression of PD-L1 and stem/biliary markers in HCC. Expression of PD-L1 (a), and stem/biliary markers; EpCAM (b), CK19 (c), and SALL4 (d) by immunohistochemistry. Scale bar, 100 $\mu \mathrm{m}$. e Seventeen of the 40 PD-L1-positive HCCs (42.5\%) showed EpCAM expression; 43 of the 114 PD-L1negative HCCs $(37.4 \%)$ were positive for EpCAM ( $p=0.5937$, Pearson's $\chi^{2}$ test). $\mathbf{f}$ In the 40 PD-L1-positive HCCs, 20 (50.0\%) were positive for CK19; only 10 of 114 PD-L1-negative HCCs (8.8\%) showed CK19 expression $\left(p<0.0001\right.$, Pearson's $\chi^{2}$ test). g Similarly, a significantly higher percentage of tumors showed expression of SALL4 in the PD-L1-positive HCCs compared to the PD-L1-negative HCCs, where 25\% (10/40) of the PD-L1-positive tumors and 9.7\% (11/114) of the PD-L1-negative tumors showed expression of SALL4, respectively ( $p=0.0149$, Pearson's $\chi^{2}$ test).

and 21 of 154 HCCs $(39.0,19.5$, and $13.6 \%$ of the total of cases), respectively (Fig. 1b-d). No relationship was observed between expressions of PD-L1 and EpCAM in HCC $(p=0.5937$; Fig. 1e). However, expressions of CK19 and SALL4 were positively associated with the expression of PD-L1 ( $p<0.0001$ and 0.0149 for CK19 and SALL4, respectively; Fig. 1f, g).

\section{Genetic Alterations and Activation of Oncogenic Pathways in PD-L1-Positive HCC}

To determine the genetic background of PD-L1-positive HCCs, we analyzed the gene mutations and amplifications of 409 types of cancer-associated genes using the comprehensive cancer panel and the TERT promoter. In total, 139/154 cases showed at least 1 mutation. The most frequent mutation was detected in the TERT promoter (64.3\%) followed by TP53 (22.7\%), CTNNB1 (22.1\%), SYNE1 (6.5\%), DST (5.8\%), and APC, ARID1A, ARID2, and CSMD3 (4.5\%) (online suppl. Fig. S1). In order to analyze the association between alterations in oncogenic pathways and PD-L1 expression in HCC, we categorized the cancer-related genes into 8 groups based on the function of the oncogenic gene products: $\beta$-catenin, p53/cell cycle, chromatin remodeling, epigenetic regulation, the phosphatidyl inositol 3-kinase (PI3K)-Akt pathway, oxidative and endoplasmic reticulum stress, the TERT promoter, and DNA repair pathways [20-23]. Genetic alterations in the $\beta$-catenin pathway were found in 39/154 HCCs (25.3\%). Similarly, 51/154 (33.1\%) contained alterations in genes related to p53/cell cycle 
Table 1. Expression of PD-L1 and genetic alteration in each oncogenic pathway in HCC

\begin{tabular}{|c|c|c|c|}
\hline Oncogenic pathway ${ }^{\mathrm{a}}$ & $\begin{array}{l}\text { PD-L1-positive HCC } \\
(n=40)\end{array}$ & $\begin{array}{l}\text { PD-L1-negative HCC } \\
(n=114)\end{array}$ & $p$ value $^{\mathrm{b}}$ \\
\hline \multicolumn{4}{|l|}{$\beta$-catenin } \\
\hline Alteration (+) & 5 & 34 & \multirow[t]{2}{*}{0.0302} \\
\hline Alteration $(-)$ & 35 & 80 & \\
\hline \multicolumn{4}{|l|}{ p53/cell cycle control } \\
\hline Alteration (+) & 15 & 37 & \multirow[t]{2}{*}{0.5617} \\
\hline Alteration (-) & 25 & 77 & \\
\hline \multicolumn{4}{|l|}{ Chromatin remodeling } \\
\hline Alteration (+) & 7 & 17 & \multirow[t]{2}{*}{0.6979} \\
\hline Alteration (-) & 33 & 97 & \\
\hline \multicolumn{4}{|l|}{ Epigenetic regulator } \\
\hline Alteration (+) & 5 & 19 & \multirow[t]{2}{*}{0.5319} \\
\hline Alteration (-) & 35 & 95 & \\
\hline \multicolumn{4}{|l|}{ PI3K-Akt } \\
\hline Alteration (+) & 8 & 8 & \multirow[t]{2}{*}{0.0206} \\
\hline Alteration (-) & 32 & 106 & \\
\hline \multicolumn{4}{|l|}{ Oxidative and ER stress } \\
\hline Alteration (+) & 3 & 12 & \multirow[t]{2}{*}{0.5786} \\
\hline Alteration $(-)$ & 37 & 102 & \\
\hline \multicolumn{4}{|l|}{ DNA repair } \\
\hline Alteration (+) & 3 & 11 & \multirow[t]{2}{*}{0.6842} \\
\hline Alteration $(-)$ & 37 & 103 & \\
\hline \multicolumn{4}{|l|}{ TERT promoter } \\
\hline Alteration (+) & 26 & 73 & \multirow[t]{2}{*}{0.9127} \\
\hline Alteration (-) & 14 & 41 & \\
\hline
\end{tabular}

Bold type denotes significance. ${ }^{a}$ The presence or absence of alteration in the oncogenic pathway was determined based on the mutations, homozygous deletion, and/or amplification of cancer-related genes in each pathway.

b Pearson's $\chi^{2}$ test.

control. The frequencies of alterations in other cancer-related pathways are as follows: $15.6 \%$ (24/164) in chromatin remodeling, $15.6 \%$ (24/154) in epigenetic regulation, $10.4 \%(16 / 154)$ in PI3K-Akt, 9.7\% (15/154) in oxidative and endoplasmic reticulum stress, 9.1\% (14/154) in DNA repair pathways, and 64.3\% (99/154) in the TERT promoter (online suppl. Fig. S2). Interestingly, genetic alterations involved in the PI3K-Akt were significantly associated with PD-L1 positivity whereas mutations in the $\beta$-catenin pathways were inversely correlated with PD-L1 in HCC. As shown in Table 1, 8/40 PD-L1-positive HCCs (8/40; 20\%) carried genetic alterations in the PI3K-Akt pathway whereas 8/114 PD-L1-negative HCCs (8/114; $7 \%$ ) showed alterations in this pathway ( $p=0.0206$ : Table 1$)$. On the other hand, mutations related to $\beta$-catenin activation were more common in PD-L1-negative tumors than in PD-L1-positive tumors where $12.5 \%$ (5/40) and 29.8\% (34/114) of the HCCs showed mutations in the $\beta$-catenin pathway among PD-L1-positive and PD-L1-negative tumors, respectively ( $p=0.0302$ : Table 1 ). The details of the specific mutations involved in the PI3K-Akt and $\beta$-catenin pathways detected in this HCC cohort are shown in online supplementary Table S3. Among the 16 HCCs with mutations in this pathway, 8 were PD-L1-positive. The majority of the mutations were inactivating mutations in the tumor suppressor PTEN (phosphatase and tensin homolog deleted from chromosome 10) or TSC1/2 (tuberous sclerosis complex) genes (nonsense mutation or deletion), and activating mutations of the PIK3CA gene (the His1047Arg mutation that was known to be functional), which could lead to the constitutive activation of 


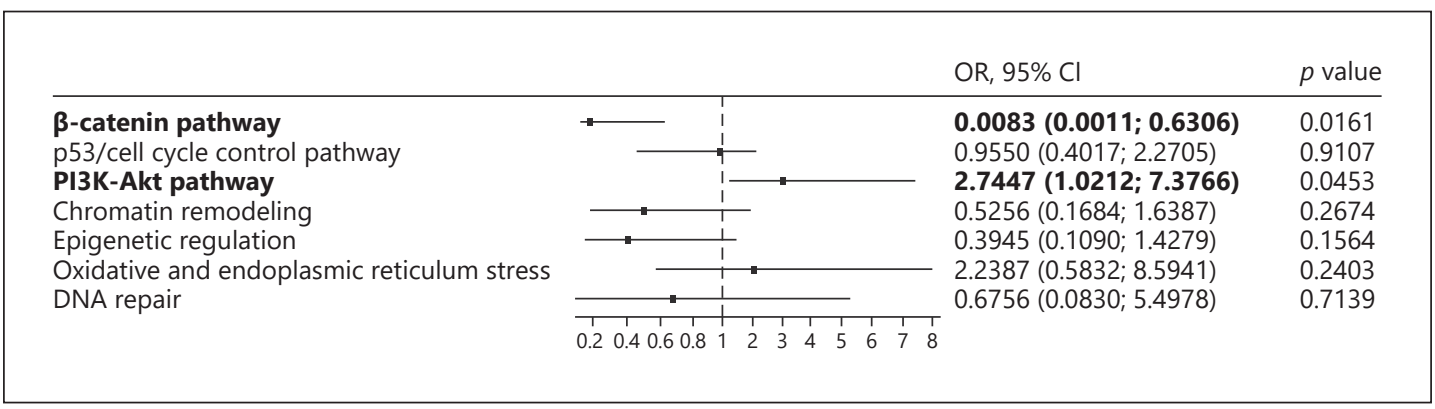

Fig. 2. The odds ratio (OR) of genetic alteration of each signaling pathway for the relative risk of expressing PD-L1 in the HCC cohort from the publicly available TCGA database. The dataset of whole exome sequence and mRNA expression (RNA sequence) of CD274 (PD-L1) in 373 HCC tissues was obtained. HCCs were classified based on the mutation involved in each oncogenic pathway, which was available in the cBioPortal for Cancer Genomics web site (https://www.cbioportal.org). We calculated the OR of genetic alterations of each oncogenic pathway for the relative risk of PD-L1 expression $\geq 35$ using logistic regression analysis. Alterations in the PI3K-Akt pathway showed the highest OR for the high expression of PD-L1 ( $p=0.0453$; OR 2.7447; 95\% confidence interval [CI] 1.0212-7.3766), whereas those in the $\beta$-catenin pathway showed the lowest risk ( $p=0.0161$; OR 0.0083; 95\% CI 0.0011-0.6306). Mutations involved in the TERT were excluded from the analysis because only 2 HCCs showed mutations based on the exome sequencing. Genes for classification of the mutations into each pathway which were included in the Ion AmpliSeq ${ }^{\text {TM }}$ Comprehensive Cancer Panel were selected from the TCGA database by referring to the gene set shown in online supplementary Figure S2. Annotation of the genes for the oncogenic pathways is listed below. $\beta$-Catenin pathway: $A P C$, AXIN1, and CTNNB1. p53/cell cycle control pathway: ATM, ATR, CCND1, CCND2, CCNE1, CDK12, CDKN2A, CREBBP, FBXW7, MDM2, RB1, RPS6KA2, and TP53. PI3K-Akt pathway: PI3KCA, PI3KC2B, PI3KCB, PTEN, MAP3K7, RPS6CA2, TSC1, and TSC2. Chromatin remodeling: ARID1A, ARID1B, ARID2, CREBBP, EZH2, and SMARCA2. Epigenetic regulation: DNMT3A, IDH1, IDH2, KMT2A, KMT2B, KMT2C, KMT2D, SETD2, TET1, and TET2. Oxidative and endoplasmic reticulum stress: ATF1, CYP2C19, KEAP1, NFE2L2, and ROS1. DNA repair: ATM, ERCC2, ERCC5, FANCA, MLH1, MSH2, MSH6, XZCC2.

the PI3K-Akt pathway [24]. On the other hand, the majority of the mutations in the $\beta$-catenin pathways were missense mutations of the CTNNB1 gene, and were mainly detected in PD-L1-negative tumors; only 5/39 HCCs with mutation in the $\beta$-catenin pathways were PD-L1-positive.

We also examined the effect alterations in the PI3K-Akt and the $\beta$-catenin pathways had on the expression of PD-L1, using the publicly available database in TCGA (TCGA cohort), where exome sequence and comprehensive expression data were available for 373 HCCs. We compared the odds ratio (OR) of the genetic alteration of each oncogenic pathway for the relative risk of expressing PD-L1. Candidate genes involved in each oncogenic pathway were selected based on mutations that were detected in HCCs from the Kindai cohort (online suppl. Fig. S2). Even in the TCGA cohort, mutations in the PI3K-Akt pathway could most positively affect the expression of PD-L1 (OR 2.7447; 95\% confidence interval [CI] 1.0212-7.3766; $p=$ $0.0453)$. On other hand, mutations in the $\beta$-catenin pathway were related to absence of PD-L1 expression (OR 0.0083; 95\% CI 0.0011-0.6306; $p=0.0161$; Fig. 2).

\section{Dense Infiltration of CD8+ Cells and the Expression of Co-Inhibitory Receptors in}

PD-L1-Positive HCCS

Two different clinical trials revealed that the expression of PD-L1 was not associated with the efficacy of anti-PD-1 antibody treatment in HCC patients, although patients with PD-L1-positive HCC tended to respond better than those without PD-L1 $[9,10]$. Therefore, we examined the infiltration of CD8+ cells in PD-L1-positive and PD-L1-negative HCC tissues 


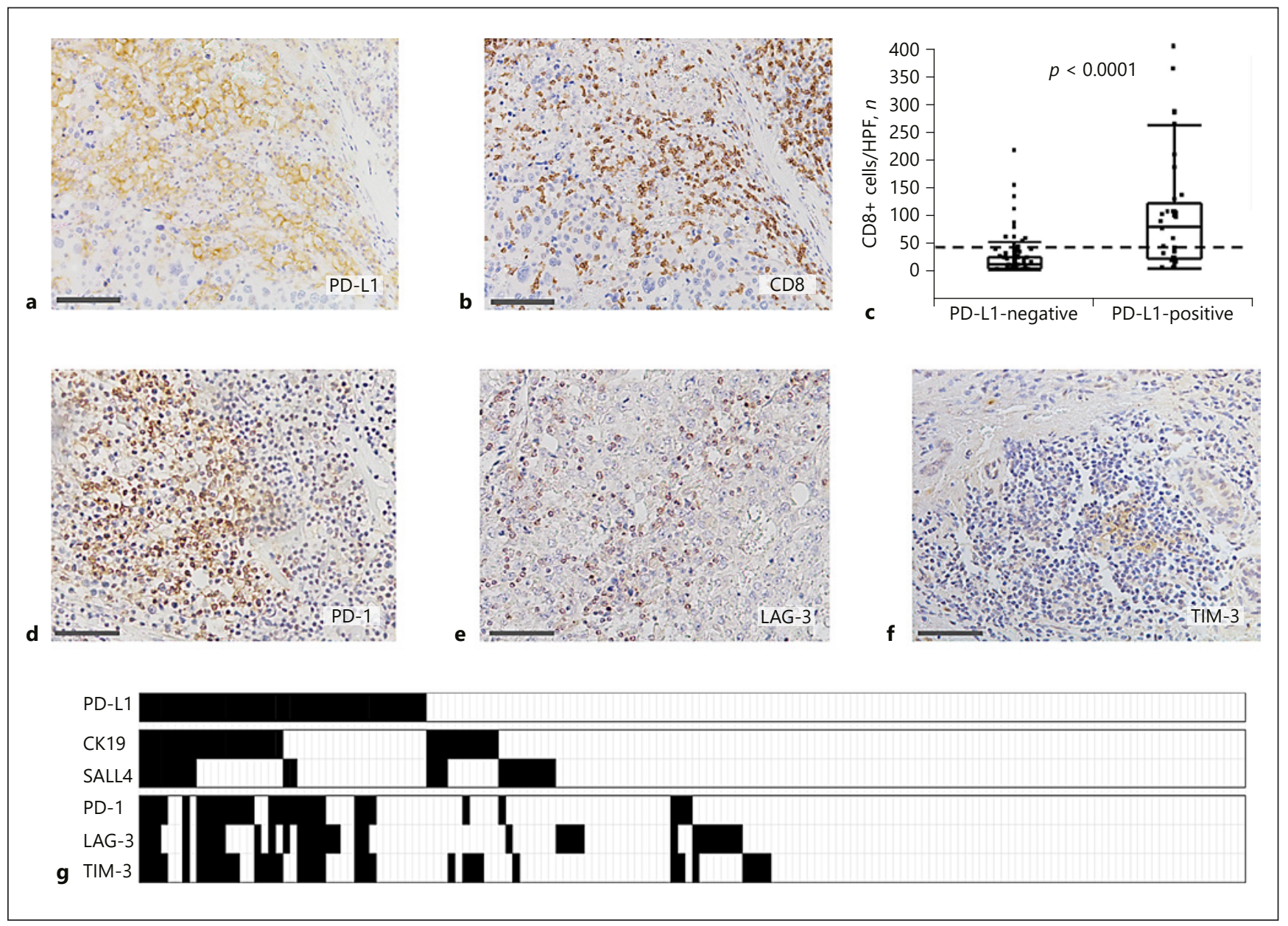

Fig. 3. Expression of co-inhibitory receptors in the CD8+-infiltrated cells in a PD-L1-positive tumor. a Membranous expression of PD-L1 in HCC tissues. b Dense infiltration of CD8+ cells in the same section of HCC tissues shown in (a). Scale bar, $100 \mu \mathrm{m}$. c Comparison of degree of infiltration of CD8+ cells in PD-L1-negative and PD-L1-positive HCCs. Numbers of CD8+ cells/HPF are shown in the vertical axis. The box-and-whisker plots denote 75 and $95 \%$ distribution, and the lines in the boxes show the median values of each group. The dashed line shows the mean value of this cohort. The median degree of infiltration of CD8+ cells was 63.7 cells/HPF (25-75 percentiles; 20.2-103.4) in PD-L1-positive and 9.4 cells/HPF (25-75 percentiles; 2.4-22.9) in PD-L1-negative HCC tissues, respectively ( $p<0.0001$, Wilcoxon sum-rank test). Expression of PD-1 (d), LAG-3 (e), and TIM-3 (f) on immunohistochemistry. Scale bar, $100 \mu \mathrm{m}$. $\mathbf{g}$ The heat map shows the association among expressions of PD-L1, stem/biliary cell markers in HCC cells, and those of co-inhibitory receptors in the tumor-infiltrated mononuclear cells (TIMCs). The black rectangle shows the cases with positive immunostaining. The expression of the stem cell markers, CK19 and SALL4, was more frequent in PD-L1-positive HCCs. Although infiltration of CD8+ cells was more prominent in PD-L1-positive HCCs (as shown in c); multiple co-inhibitory receptors were also expressed in TIMCs of PD-L1-positive HCCs.

(Fig. 3a, b). The median number of CD8+ TIMCs/high power field (HPF) was 9.4 (25-75 percentiles: 2.4-22.9) in PD-L1-negative HCC and 63.7 (25-75 percentiles: 20.2-103.4) in PD-L1-positive ( $p<0.0001$ by Wilcoxon sum-rank test; Fig. 3c).

Next, we analyzed the expression of the other co-inhibitory receptors LAG-3 and TIM-3 in TIMCs, in addition to PD-1 (Fig. 3d-f). PD-1 was detected in TIMCs in 60\% (24/40) of PD-L1-positive HCC cases, whereas only 4.4\% (5/114) of PD-L1-negative HCC showed PD-1 expression on TIMCs $\left(p<0.0001\right.$, Pearson's $\chi^{2}$ test; online suppl. Table S4). Similarly, LAG- 


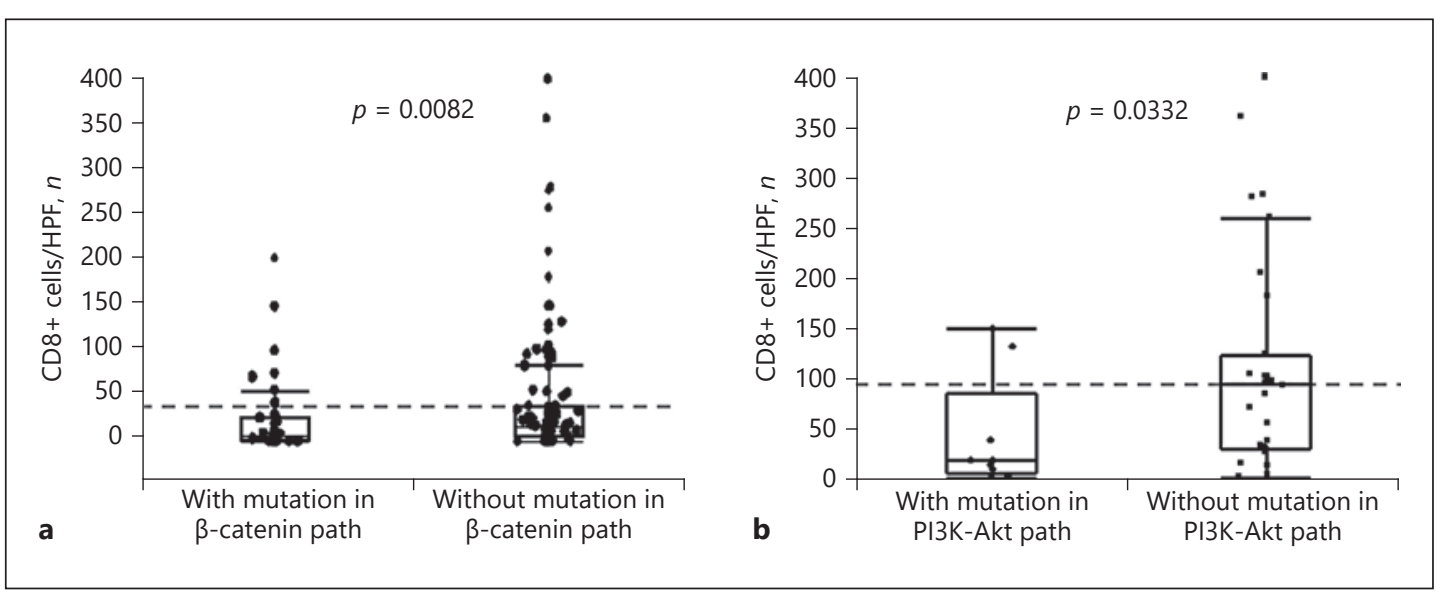

Fig. 4. Infiltration of CD8+ cells in tumors and genetic alterations in oncogenic pathways. a The degree of CD8+ TIMCs and the presence of activating mutations in the $\beta$-catenin pathway (path). The median degree of infiltration of CD8+ cells was 6.2 cells/HPF (25-75 percentiles; $1.3-26.4$ ) in tumors with mutation and 17.6 cells/HPF (25-75 percentiles; 5.8-38.0) in tumors without mutation in the $\beta$-catenin pathway, respectively ( $p=0.0082$, Wilcoxon sum-rank test). $\mathbf{b}$ The degree of CD8+ TIMCs and the presence of activating mutations in the PI3K-Akt pathway (path) in PD-L1-positive HCCs. The median number of CD8+ cells (25th-75th percentile) was 20.3 (12.5-36.4) in the PD-L1-positive HCCs with mutations in the PI3K-Akt pathway and 94.3 (30.5-119.7) in those without mutations, respectively ( $p=0.0332$, Wilcoxon sum-rank test).

Table 2. Comparisons of number of co-inhibitory receptors expressed in tumor-infiltrated mononuclear cells in PD-L1-positive and negative HCCs

\begin{tabular}{|c|c|c|c|c|}
\hline \multirow[t]{2}{*}{ HCC type } & \multicolumn{4}{|c|}{ Number of co-inhibitory receptors expressed in TIMCs } \\
\hline & 3 & 2 & 1 & 0 \\
\hline PD-L1-positive $(n=40)$ & $15(37.5)$ & $7(17.5)$ & $6(15.0)$ & $12(30.0)$ \\
\hline PD-L1-negative $(n=114)$ & $1(0.9)$ & $3(2.6)$ & $21(18.4)$ & $89(78.1)$ \\
\hline
\end{tabular}

Values express $n$ (\%). $p<0.0001$, Pearson's $\chi^{2}$ test. TIMCs, tumor-infiltrated mononuclear cells.

3-positive and TIM-3-positive TIMCs were more frequently observed in PD-L1-positive HCCs than in PD-L1-negative HCCs ( $p<0.0001$ for both LAG-3 and TIM-3, Pearson's $\chi^{2}$ test; online suppl. Table S4). In PD-L1-positive HCCs, 37.5\% (15/40) of the cases expressed 3 kinds of co-inhibitory receptors on TIMCs and 17.5\% (7/40) expressed 2 kinds of co-inhibitory receptors. Conversely, only $0.9 \%(1 / 114)$ and $2.6 \%(3 / 114)$ of the PD-L1-negative HCCs expressed 3 and 2 kinds of co-inhibitory receptors on TIMCs, respectively $(p<0.0001$, Pearson's $\chi^{2}$ test; Table 2; Fig. 3g). The concomitant staining of CD8+ and co-inhibitory receptors revealed that PD-1, TIM-3, and LAG-3 were mainly detected on CD8+ T cells (online suppl. Fig. S3). This evidence suggests that more than half of PD-L1-positive HCCs contain CD8+ TIMCs that express multiple co-inhibitory receptors.

\section{Inflamed and Non-Inflamed Tumors and Genetic Alterations in HCC}

Because the expression of PD-L1 in HCC was associated with the degree of infiltration of CD8+ cells as well as genetic alterations in the PI3K-Akt and the $\beta$-catenin pathways, we compared the number of CD8+ TIMCs in HCCs with and without such genetic alterations. As 
Table 3. Summary of PD-L1 expression and molecular and immunological background of HCCs

\begin{tabular}{|c|c|c|c|c|}
\hline $\begin{array}{l}\text { Expression } \\
\text { of PD-L1 }\end{array}$ & $\begin{array}{l}\text { Expression of } \\
\text { stem/biliary } \\
\text { cell markers }^{\mathrm{a}}\end{array}$ & $\begin{array}{l}\text { Alteration in } \\
\text { oncogenic } \\
\text { pathway }\end{array}$ & $\begin{array}{l}\text { Degree of infiltration } \\
\left.\text { (CD8 }^{+} \text {TIMCs/HPF }\right)^{\mathrm{b}}\end{array}$ & $\begin{array}{l}\text { Cases with expression } \\
\text { of multiple co-inhibitory } \\
\text { receptors }^{\mathrm{c}}, \mathrm{n} / \mathrm{N}(\%)\end{array}$ \\
\hline $\begin{array}{l}\text { Positive } \\
(n=40)\end{array}$ & frequent: 55\% & $\begin{array}{l}\text { with PI3K-Akt } \\
\text { without PI3K-Akt }\end{array}$ & $\begin{array}{l}\text { low }(20.3) \\
\text { high }(94.3)\end{array}$ & $\begin{array}{l}3 / 8(37.5) \\
19 / 32(59.3)\end{array}$ \\
\hline $\begin{array}{l}\text { Negative } \\
(n=114)\end{array}$ & rare: $16.7 \%$ & $\begin{array}{l}\text { with } \beta \text {-catenin } \\
\text { without } \beta \text {-catenin }\end{array}$ & $\begin{array}{l}\text { very low (5.8) } \\
\text { low (12.1) }\end{array}$ & $\begin{array}{l}1 / 34(2.9) \\
3 / 80(3.8)\end{array}$ \\
\hline
\end{tabular}

shown in Figure 4a, the presence of mutations in the $\beta$-catenin pathway were associated with a low degree of infiltration of CD8+ cells ( $p=0.0082$ by Wilcoxon sum-rank test). We also compared the degree of CD8+ TIMCs with and without mutations in the $\beta$-catenin pathway only in PD-L1-negative HCCs, where the infiltration of CD8+ cells was not prominent. Although not statistically significant, the degree of CD8+ TIMCs was lower in tumors with mutations in the $\beta$-catenin pathway than in those without mutations, even among PD-L1-negative HCC cases (median number of CD8+ cells: 5.8 vs. 12.1 in HCCs with mutations in the $\beta$-catenin pathway and those without mutations; $p=0.0525$ by Wilcoxon sum-rank test; online suppl. Fig. S4). On the other hand, the majority of PD-L1-positive HCCs contained many CD8+ TIMCs (Fig. 3c), suggesting that inflammatory cytokines secreted by CD8+ cells, such as IFN- $\gamma$, may induce PD-L1 expression in HCC cells. However, a subset of PD-L1-positive HCCs lacked dense infiltration of CD8+ cells. Because genetic alterations in cancer cells may also be attributed to PD-L1 expression without stimulation of extrinsic cytokines, and mutations in the PI3K-Akt pathway are positively associated with PD-L1 expression (Table 1), we compared the degree of CD8+ TIMCs between tumors with mutations in the PI3K-Akt pathway and those without mutations among PD-L1-positive cases. The median number of CD8+ cells (25th-75th percentile) was 20.3 (12.5-36.4) in the PD-L1-positive HCCs with mutations in the PI3K-Akt, and $94.3(30.5-119.7)$ in the HCCs without mutations ( $p=0.0332$, Wilcoxon sum-rank test; Fig. 4b).

\section{Discussion}

Several targeted therapeutic agents have become available for the treatment of HCC. However, acquired mutations in HCC could result in the development of resistance to such treatments, so other types of therapies are required in these cases $[1,6]$. Recently, anti-PD-1 antibody became one of the key agents for many types of malignancy [12]. The objective response rate to anti-PD-1 antibody therapy is around 20\% in HCC $[9,10]$, and many recent clinical trials are combining anti-PD-1 antibody with other types of targeted therapeutic agents [25]. This clinical evidence prompted us to ask important questions. Do the genetic backgrounds of HCC affect the immunological status of HCC? What type of therapeutic agent is ideal to combine with anti-PD-1/PD-L1 therapy based on the background immunological microenvironment? To answer these questions, we conducted a comprehensive analysis of immune checkpoint protein expressions and genetic alterations using a large number of human HCC samples. 
Consistent with previous reports, PD-L1-positive HCC shared characteristics with highgrade tumors $[26,27]$. Although the detailed mechanism of PD-L1 expression in HCC is still controversial, environmental factors that promote the development of high-grade tumors, such as immunogenicity in advanced HCC, should give rise to the expression of PD-L1 via the induction of IFN $-\gamma$ from CD8+ cells $[28,29]$. On the other hand, Calderaro et al. [30] reported that PD-L1 is expressed in the progenitor subtype of CK19-positive HCC. Kurebayashi et al. [31] also demonstrated a positive association between the expression of PD-L1 and stem/ biliary cell markers. We also found a significant increase of PD-L1 expression in CK19- and/ or SALL4-positive HCCs compared to those without such markers. From this point of view, anti-PD-1 and PD-L1 antibodies could be considered as key therapeutic agents for HCC with stem cell-like characteristics (which is resistant to conventional therapy).

Next, we analyzed the status of CD8+ cells to determine the degree of immune cell activity in HCC tissues. As shown in Figure 3, the infiltration of CD8+ cells was significantly increased in PD-L1-positive compared to PD-L1-negative HCC. However, we also found multiple co-inhibitory receptors expressed in CD8+ TIMCs, suggesting that the majority of CD8+ cells in PD-L1-positive tumor tissues were exhausted immune cells. Therefore, interventions targeting the PD-1/PD-L1 axis might be insufficient for an antitumor effect in such cases [32]. Blockade of LAG-3 or TIM-3, in addition to anti-PD-1/PD-L1 antibody, would be necessary for the restoration of $\mathrm{T}$ cell function [33].

Conversely, it has been reported that some HCCs lacking PD-L1 expression could also respond to anti-PD-1 antibody [9]. Based on our findings that showed an association between the expression of PD-L1 and stem/biliary cell markers, it could be that anti-PD-1/PD-L1 antibody has the potential to target cancer stem cells (CSCs). Generally, the population of CSCs is too small to be detected on IHC; such tumors might be judged as PD-L1-negative. In this case, HCCs might respond to PD-1/PD-L1 blockade because mature cancer cells, in which PD-L1 expression was downregulated during differentiation, may originate from PD-L1-positive CSCs that are sensitive to anti-PD-1 antibody. A previous report that showed PD-L1 accumulation on CSCs also supported this idea [34].

We sought to elucidate the mutational profile of PD-L1-positive HCCs since alterations in the $P D-L 1$ gene that induce PD-L1 expression, such as gene amplification and deletion of the 3'-untranslated region, have not previously been reported in HCCs [35]. As PD-L1 expression can be regulated by mitogen-activated protein kinase and PI3K-Akt signaling [29], we specifically focused on any alterations in these signaling pathways [36]. Interestingly, the genetic alterations involved in the PI3K-Akt pathway were more frequently detected in PD-L1-positive tumors than in PD-L1-negative tumors. We confirmed this result using the TCGA database; mutations in the PI3K-Akt pathway could mostly affect the increased expression of PD-L1 among the alterations of oncogenic pathways (Fig. 2). Reportedly, the inactivation of PTEN, which is known to suppress PI3K, was associated with the expression of PD-L1 in glioma [37]. More importantly, a recent report suggested that inactivating the mutation of PTEN and activating that of PI3K were associated with the expression of CK19 in HCC [38], where PD-L1 expression was frequent. In addition, the activation of the PI3K-Akt pathway is one of the characteristics of CSCs [39]. Therefore, genetic alterations and constitutive activation of the PI3K-Akt pathway could also give rise to the overexpression of PD-L1 and induce stem cell feature in HCCs.

Previous reports identified the immune-specific subclass of HCC, where molecular subclass S1 was predominant [40,41]. As shown above, dense infiltration of CD8+ cells is one of the characteristics of PD-L1-positive HCC, suggesting that this type of HCC could, at least partially, correspond to an immune-specific subclass [41]. On the other hand, it was reported that molecular subclass S2, where the activation of PI3K-Akt is more common, is mainly classified as a non-immune subclass [41]. In this study, we found that infiltration of CD8+ cells 
was not prominent in PD-L1-positive HCCs with mutation in the PI3K-Akt pathway, compared to those without mutation. Therefore, constitutive activation of the PI3K-Akt pathway in HCC could induce the expression of PD-L1, even in a non-inflamed background, where environmental stimulators such as IFN- $\gamma$ might not play a major role in the induction of PD-L1.

On the other hand, activation of the $\beta$-catenin pathway by mutations was more common in PD-L1-negative HCC; this finding was confirmed in the TCGA cohort. Previous reports show that the activation of $\beta$-catenin could lead to the downregulation of $\mathrm{C}$ - $\mathrm{C}$ motif chemokine ligand 4 and block the infiltration of $T$ cells into the tumor in melanoma cases $[42,43]$. We also found that the activating mutation of the $\beta$-catenin pathway was inversely correlated with the degree of CD8+ TIMCs, suggesting that a lack of IFN- $\gamma$ in immune cells should give rise to low expression of PD-L1 in HCC with mutations in the $\beta$-catenin pathway.

In this study, we found unique characteristics of tumors in the context of PD-L1 expression that showed an important clue for the management of HCC using immune checkpoint inhibitors (Table 3). First, advanced HCCs, especially those with stem/biliary cell markers, showed increased PD-L1 expression, suggesting that PD-1/PD-L1 blockade may be a promising therapeutic approach for such refractory tumors. However, as multiple co-inhibitory receptors are observed in CD8+ cells in tumor tissues (Table 3), the combination with other types of immune checkpoint inhibitors such as anti-TIM-3 and anti-LAG-3 antibodies may be required for the treatment of HCC containing exhausted CD8+ cells [44]. On the other hand, a subset of PD-L1-positive HCCs showed activated mutation in the PI3K-Akt pathway and a relatively small number of exhausted CD8+ TIMCs. In this case, monotherapy of anti-PD-1/PD-L1, or the inhibition of the PI3K-Akt pathway (that leads to a reduction in PD-L1), could restore antitumor immunity. Indeed, a previous study showed that inhibition of PI3K downregulated PD-L1 expression and enhanced the antiproliferative effect of IFN- $\gamma$ in lung cancer [45].

More importantly, the PI3K-Akt pathway is frequently activated in CSCs and PI3K inhibitors preferentially target CSCs [39]. As the expression of stem/biliary cell markers in HCC is associated with PD-L1 expression and anti-PD-1/PD-L1 antibody might also target CSCs, the dual blockade of the PD1/PD-L1 axis and the PI3K-Akt pathway could prove to be an attractive option for refractory HCC patients [46]. A recent report suggested that HCCs with genetic alterations in the PI3K-Akt pathway showed a worse outcome in patients on conventional TKI treatment [23]. The development of a novel combination therapy for this type of HCC is thus critical. On the other hand, PD-L1-negative HCCs frequently showed activating mutations in the $\beta$-catenin pathway and a lack of CD8+ TIMCs (Table 3). Another recent report showed that genetic alterations of the $\beta$-catenin pathway were associated with innate resistance to immune checkpoint inhibitors [23]. Blockade of $\beta$-catenin could be therefore be worth taking into consideration in cases with an absence of PD-L1 expression as well as infiltration of CD8+ cells.

Despite the detailed analyses of HCC tissues, we did not show clinical data regarding the efficacy of immune checkpoint inhibitors in the context of PD-L1 and stem/biliary cell marker expression. However, several clinical trials that combine anti-PD-1/PD-L1 antibody with other agents, such as anti-TIM-3 antibody, anti-LAG-3 antibody, and PI3K inhibitors, are ongoing. Therefore, the data presented here may be informative for the development of novel therapeutic strategies using immune checkpoint inhibitors for the treatment of refractory HCC.

\section{Statement of Ethics}

We used archived tissue samples for this study that were obtained with patient consent at Kindai University Hospital. The study was approved by the ethics committee of Kindai University Hospital. The study protocol conforms to the ethics guidelines of the 1975 Declaration of Helsinki. 


\begin{tabular}{l|l|}
\hline Liver Cancer 2020;9:426-439 \\
\hline DOI: 10.1159/000506352 & $\begin{array}{l}\text { @ 2020 The Author(s). Published by S. Karger AG, Basel } \\
\text { www.karger.com/lic }\end{array}$ \\
\hline
\end{tabular}

Nishida et al.: Immune Status and Mutations in HCC

\section{Disclosure Statement}

N.N. received research grants from Gilead Sciences. M.K. received research grants from Taiho Pharmaceuticals, Chugai Pharmaceuticals, Otsuka, Takeda, Sumitomo Dainippon-Sumitomo, Daiichi Sankyo, AbbVie, Astellas Pharma, and Bristol-Myers Squibb; grants and personal fees from MSD, Eisai, and Bayer; and is an adviser for MSD, Eisai, Bayer, Bristol-Myers Squibb, Eli Lilly, and ONO Pharmaceutical. All other authors have nothing to declare.

\section{Funding Sources}

This work was supported in part by a Grant-in-Aid for Scientific Research from the Japan Society for the Promotion of Science (KAKENHI) (16K09382 to N.N. and 18K07922 to M.K.) and a grant from the Smoking Research Foundation (N.N.).

\section{Author Contributions}

Study concept and design: N.N. Acquisition of data: N.N., K.S., T.A., M. Takenaka, S.H., Y.K., M. Takita, Y.M., H.I., and K.U. Analysis and interpretation of the data: N.N., K.S., K.N., M.K. Drafting of the manuscript: N.N. Revision of the manuscript for intellectual content: all authors. Statistical analysis: N.N. Study supervision: N.N. and M.K.

\section{References}

1 Llovet JM, Montal R, Sia D, Finn RS. Molecular therapies and precision medicine for hepatocellular carcinoma. Nat Rev Clin Oncol. 2018 Oct;15(10):599-616.

2 Villanueva A. Hepatocellular Carcinoma. N Engl J Med. 2019 Apr;380(15):1450-62.

3 Terris B, Cavard C, Perret C. EpCAM, a new marker for cancer stem cells in hepatocellular carcinoma. J Hepatol. 2010 Feb;52(2):280-1.

4 Yong KJ, Gao C, Lim JS, Yan B, Yang H, Dimitrov T, et al. Oncofetal gene SALL4 in aggressive hepatocellular carcinoma. N Engl J Med. 2013 Jun;368(24):2266-76.

5 Miltiadous O, Sia D, Hoshida Y, Fiel MI, Harrington AN, Thung SN, et al. Progenitor cell markers predict outcome of patients with hepatocellular carcinoma beyond Milan criteria undergoing liver transplantation. J Hepatol. 2015 Dec;63(6):1368-77.

6 Nishida N, Kitano M, Sakurai T, Kudo M. Molecular Mechanism and Prediction of Sorafenib Chemoresistance in Human Hepatocellular Carcinoma. Dig Dis. 2015 Oct;33(6):771-9.

7 Nishida N, Kudo M. Clinical Significance of Epigenetic Alterations in Human Hepatocellular Carcinoma and Its Association with Genetic Mutations. Dig Dis. 2016;34(6):708-13.

8 Nishida N, Nishimura T, Kaido T, Minaga K, Yamao K, Kamata K, et al. Molecular Scoring of Hepatocellular Carcinoma for Predicting Metastatic Recurrence and Requirements of Systemic Chemotherapy. Cancers (Basel). 2018 Sep;10(10):E367.

9 El-Khoueiry AB, Sangro B, Yau T, Crocenzi TS, Kudo M, Hsu C, et al. Nivolumab in patients with advanced hepatocellular carcinoma (CheckMate 040): an open-label, non-comparative, phase 1/2 dose escalation and expansion trial. Lancet. 2017 Jun;389(10088):2492-502.

10 Zhu AX, Finn RS, Edeline J, Cattan S, Ogasawara S, Palmer D, et al.; KEYNOTE-224 investigators. Pembrolizumab in patients with advanced hepatocellular carcinoma previously treated with sorafenib (KEYNOTE224): a non-randomised, open-label phase 2 trial. Lancet Oncol. 2018 Jul;19(7):940-52.

11 Nishida N, Kudo M. Immune checkpoint blockade for the treatment of human hepatocellular carcinoma. Hepatol Res. 2018 Jul;48(8):622-34.

12 Taube JM, Klein A, Brahmer JR, Xu H, Pan X, Kim JH, et al. Association of PD-1, PD-1 ligands, and other features of the tumor immune microenvironment with response to anti-PD-1 therapy. Clin Cancer Res. 2014 Oct; 20(19):5064-74.

13 Nishida N, Kudo M. Oncogenic Signal and Tumor Microenvironment in Hepatocellular Carcinoma. Oncology. 2017;93 Suppl 1:160-4.

14 Yan W, Liu X, Ma H, Zhang H, Song X, Gao L, et al. Tim-3 fosters HCC development by enhancing TGF- $\beta$-mediated alternative activation of macrophages. Gut. 2015 0ct;64(10):1593-604.

15 Kim HR, Ha SJ, Hong MH, Heo SJ, Koh YW, Choi EC, et al. PD-L1 expression on immune cells, but not on tumor cells, is a favorable prognostic factor for head and neck cancer patients. Sci Rep. 2016 Nov;6(1):36956.

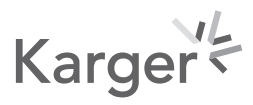


16 Tsujikawa H, Masugi Y, Yamazaki K, Itano O, Kitagawa Y, Sakamoto M. Immunohistochemical molecular analysis indicates hepatocellular carcinoma subgroups that reflect tumor aggressiveness. Hum Pathol. 2016 Apr;50:24-33.

17 Sato Y, Kinoshita M, Takemura S, Tanaka S, Hamano G, Nakamori S, et al. The PD-1/PD-L1 axis may be aberrantly activated in occupational cholangiocarcinoma. Pathol Int. 2017 Mar;67(3):163-70.

18 Wu J, Lin G, Zhu Y, Zhang H, Shi G, Shen Y, et al. Low TIM3 expression indicates poor prognosis of metastatic prostate cancer and acts as an independent predictor of castration resistant status. Sci Rep. 2017 Aug;7(1): 8869.

19 Nault JC, Mallet M, Pilati C, Calderaro J, Bioulac-Sage P, Laurent C, et al. High frequency of telomerase reversetranscriptase promoter somatic mutations in hepatocellular carcinoma and preneoplastic lesions. Nat Commun. 2013;4(1):2218.

20 Guichard C, Amaddeo G, Imbeaud S, Ladeiro Y, Pelletier L, Maad IB, et al. Integrated analysis of somatic mutations and focal copy-number changes identifies key genes and pathways in hepatocellular carcinoma. Nat Genet. 2012 May;44(6):694-8.

21 Totoki Y, Tatsuno K, Covington KR, Ueda H, Creighton CJ, Kato M, et al. Trans-ancestry mutational landscape of hepatocellular carcinoma genomes. Nat Genet. 2014 Dec;46(12):1267-73.

22 Schulze K, Imbeaud S, Letouzé E, Alexandrov LB, Calderaro J, Rebouissou S, et al. Exome sequencing of hepatocellular carcinomas identifies new mutational signatures and potential therapeutic targets. Nat Genet. 2015 May;47(5):505-11.

23 Harding JJ, Nandakumar S, Armenia J, Khalil DN, Albano M, Ly M, et al. Prospective Genotyping of Hepatocellular Carcinoma: Clinical Implications of Next-Generation Sequencing for Matching Patients to Targeted and Immune Therapies. Clin Cancer Res. 2019 Apr;25(7):2116-26.

24 Lindhurst MJ, Parker VE, Payne F, Sapp JC, Rudge S, Harris J, et al. Mosaic overgrowth with fibroadipose hyperplasia is caused by somatic activating mutations in PIK3CA. Nat Genet. 2012 Jun;44(8):928-33.

25 Kudo M. Systemic Therapy for Hepatocellular Carcinoma: latest Advances. Cancers (Basel). 2018 Oct; 10(11):E412.

26 Umemoto Y,OkanoS, Matsumoto Y, Nakagawara H, Matono R, Yoshiya S, et al. Prognostic impact of programmed cell death 1 ligand 1 expression in human leukocyte antigen class I-positive hepatocellular carcinoma after curative hepatectomy. J Gastroenterol. 2015 Jan;50(1):65-75.

27 Jung HI, Jeong D, Ji S, Ahn TS, Bae SH, Chin S, et al. Overexpression of PD-L1 and PD-L2 Is Associated with Poor Prognosis in Patients with Hepatocellular Carcinoma. Cancer Res Treat. 2017 Jan;49(1):246-54.

28 Prieto J, Melero I, Sangro B. Immunological landscape and immunotherapy of hepatocellular carcinoma. Nat Rev Gastroenterol Hepatol. 2015 Dec;12(12):681-700.

29 Chen J, Jiang CC, Jin L, Zhang XD. Regulation of PD-L1: a novel role of pro-survival signalling in cancer. Ann Oncol. 2016 Mar;27(3):409-16.

30 Calderaro J, Rousseau B, Amaddeo G, Mercey M, Charpy C, Costentin C, et al. Programmed death ligand 1 expression in hepatocellular carcinoma: relationship with clinical and pathological features. Hepatology. 2016 Dec;64(6):2038-46.

31 Kurebayashi Y, Ojima H, Tsujikawa H, Kubota N, Maehara J, Abe Y, et al. Landscape of immune microenvironment in hepatocellular carcinoma and its additional impact on histological and molecular classification. Hepatology. 2018 Sep;68(3):1025-41.

32 Yarchoan M, Xing D, Luan L, Xu H, Sharma RB, Popovic A, et al. Characterization of the Immune Microenvironment in Hepatocellular Carcinoma. Clin Cancer Res. 2017 Dec;23(23):7333-9.

33 Zhou G, Sprengers D, Boor PP, Doukas M, Schutz H, Mancham S, et al. Antibodies Against Immune Checkpoint Molecules Restore Functions of Tumor-Infiltrating T Cells in Hepatocellular Carcinomas. Gastroenterology. 2017 Oct;153(4):1107-1119.e10.

34 Hsu JM, Xia W, Hsu YH, Chan LC, Yu WH, Cha JH, et al. STT3-dependent PD-L1 accumulation on cancer stem cells promotes immune evasion. Nat Commun. 2018 May;9(1):1908.

35 Kataoka K, Shiraishi Y, Takeda Y, Sakata S, Matsumoto M, Nagano S, et al. Aberrant PD-L1 expression through 3'-UTR disruption in multiple cancers. Nature. 2016 Jun;534(7607):402-6.

36 Jiang X, Zhou J, Giobbie-Hurder A, Wargo J, Hodi FS. The activation of MAPK in melanoma cells resistant to BRAF inhibition promotes PD-L1 expression that is reversible by MEK and PI3K inhibition. Clin Cancer Res. 2013 Feb;19(3):598-609.

37 Parsa AT, Waldron JS, Panner A, Crane CA, Parney IF, Barry JJ, et al. Loss of tumor suppressor PTEN function increases B7-H1 expression and immunoresistance in glioma. Nat Med. 2007 Jan;13(1):84-8.

38 Chen D, Li Z, Cheng Q, Wang Y, Qian L, Gao J, et al. Genetic alterations and expression of PTEN and its relationship with cancer stem cell markers to investigate pathogenesis and to evaluate prognosis in hepatocellular carcinoma. J Clin Pathol. 2019 Sep;72(9):588-96.

39 Zhou H, Yu C, Kong L, Xu X, Yan J, Li Y, et al. B591, a novel specific pan-PI3K inhibitor, preferentially targets cancer stem cells. Oncogene. 2019 May;38(18):3371-86.

40 Hoshida Y, Nijman SM, Kobayashi M, Chan JA, Brunet JP, Chiang DY, et al. Integrative transcriptome analysis reveals common molecular subclasses of human hepatocellular carcinoma. Cancer Res. 2009 Sep;69(18): 7385-92. 
41 Sia D, Jiao Y, Martinez-Quetglas I, Kuchuk O, Villacorta-Martin C, Castro de Moura M, et al. Identification of an Immune-specific Class of Hepatocellular Carcinoma, Based on Molecular Features. Gastroenterology. 2017 Sep;153(3):812-26.

42 Spranger S, Bao R, Gajewski TF. Melanoma-intrinsic $\beta$-catenin signalling prevents anti-tumour immunity. Nature. 2015 Jul;523(7559):231-5.

43 Xue G, Romano E, Massi D, Mandalà M. Wnt/ $\beta$-catenin signaling in melanoma: preclinical rationale and novel therapeutic insights. Cancer Treat Rev. 2016 Sep;49:1-12.

44 Kim HD, Song GW, Park S, Jung MK, Kim MH, Kang HJ, et al. Association Between Expression Level of PD1 by Tumor-Infiltrating CD8+ T Cells and Features of Hepatocellular Carcinoma. Gastroenterology. 2018 Dec; 155(6):1936-1950.e17.

45 Gao Y, Yang J, Cai Y, Fu S, Zhang N, Fu X, et al. IFN- $\gamma$-mediated inhibition of lung cancer correlates with PD-L1 expression and is regulated by PI3K-AKT signaling. Int J Cancer. 2018 Aug;143(4):931-43.

46 Li H, Li X, Liu S, Guo L, Zhang B, Zhang J, et al. Programmed cell death-1 (PD-1) checkpoint blockade in combination with a mammalian target of rapamycin inhibitor restrains hepatocellular carcinoma growth induced by hepatoma cell-intrinsic PD-1. Hepatology. 2017 Dec;66(6):1920-33. 